\title{
On Analytical Solution of a Plasma Flow over a Moving Plate under the Effect of an Applied Magnetic Field
}

\author{
Taha Zakaraia Abdel Wahid ${ }^{1}{ }^{1}$ and Adel M. Morad ${ }^{1,2}$ \\ ${ }^{1}$ Department of Mathematics and Computer Science, Faculty of Science, Menoufia University, Shebin El-Kom 32511, Egypt \\ ${ }^{2}$ Department of Computational Mathematics and Mathematical Physics, Institute of Mathematics, Mechanics and Computer Science, \\ Southern Federal University, Rostov on Don 344090, Russia
}

Correspondence should be addressed to Taha Zakaraia Abdel Wahid; taha_zakaraia@yahoo.com

Received 10 July 2020; Accepted 29 September 2020; Published 22 October 2020

Academic Editor: Xin Yu

Copyright (c) 2020 Taha Zakaraia Abdel Wahid and Adel M. Morad. This is an open access article distributed under the Creative Commons Attribution License, which permits unrestricted use, distribution, and reproduction in any medium, provided the original work is properly cited.

\begin{abstract}
Our objective of this investigation is to mainly focus on the behavior of a plasma gas that is bounded by a moving rigid flat plate; its motion is damping with time. The effects of an external magnetic field on the electrons collected with each other, with positive ions, and with neutral atoms in the plasma fluid are studied. The BGK type of the Boltzmann kinetic equation is used to study the gas dynamics various regimes with Maxwellian velocity distribution functions. An analytical solution of the model equations for the unsteady flow was given using the moment and the traveling wave methods. The manner of the mean velocity of plasmas is illustrated, which is compatible with the variation of the shear stress, viscosity coefficient, and the initial and boundary conditions. Besides, the thermodynamic prediction is investigated by applying irreversible thermodynamic principles and extended Gibbs formula. Finally, qualitative agreements with previous related papers were demonstrated using 3-dimensional graphics for calculating the variables. The significance of this study is due to its vast applications in numerous fields such as in physics, engineering, commercial, and industrial applications.
\end{abstract}

\section{Introduction}

From basic research in plasma science to manufacturing, rapid developments in that field have often been preceded by revolutions in new technologies, such as lowtemperature plasmas, or novel applications, such as plasma medicine, plasma biological systems, and microelectronics [1]. For example, Miller et al. [2] have used the nonequilibrium plasma to induce immunogenic cell death in tumors as a therapeutic way for diseases of body systems exposed to the plasma. Plasma-surface interactions have gained immense interest in the last few decades in the context of promising industrial applications in the world's global commercial products, manufacturing processes, and, more recently, microelectronics, medical, and biotechnologies, which are discussed elsewhere in [3].

It is well known that there are two kinetic methods for mathematical modeling of the behavior of a particle population. These methods deal with differential equations that describe the variations of the phase space distribution function $\varphi(t, \mathbf{r}, \mathbf{v})$.. In the case of collisionless plasma, this model equation is the "Vlasov-equation" [4]. In the collisional case, Boltzmann's kinetic equation describes the collisional plasma motion considering microscopic effects. To treat the difficulty of studying the collision term in Boltzmann's kinetic equation, we should use approximate modeling. One of the essential approximate models is the Bhatnagar-GrossKrook (BGK) model, which saves the computational cost of the collision term of Boltzmann's kinetic equation [5].

Many theoretical and numerical contributions are interested in developing the methods to obtain reasonable solutions to the Boltzmann equation [6, 7]. Also, many approximations have suggested solving the kinetic equation based on the moment method [8-10]. For larger values of Knudsen number (Kn), the Boltzmann kinetic equations and the popular methods depend on the kinetic theory that has to be applied [8-10]. The high magnitude of Kn occurs in rarified gases for the high values of the mean free path or 
of the minimal value of the identified length that occurs in the micro-electro-mechanical system (MEMS) or nanoelectro-mechanical system (NEMS) devices [11-13]. That property donates the Boltzmann equation a fantastic advantage and many commercial applications [14, 15]. Several papers were treated with Boltzmann equation and its applications in many physical severe situations such as thermal field and microgas sensor $[16,17]$, irreversible thermodynamics and plasma with kinetic regime [18-22], oscillating flow $[23,24]$, thermal radiation [25-27], plasma [28], ultrarelativistic heavy-ion collisions [29], photon gas [30], granular fluids [31], and electron energy distribution function [32, 33].

In the Chapman-Enskog method, transport equations are constructed at successive levels of approximation by expanding the distribution function for the parameters around the equilibrium distribution function, while in Grad's method, the transport equations for macroscopic molecular averages are carried out by taking velocity moments of the distribution function, which is approximated by an expansion in orthonormal polynomials [34, 35].

Interaction between moving plasmas and solid surfaces in modern technological applications has a rich history throughout plasma technologies. For example, Chang and Chang [36] have studied the kinetic problem for plasma boundary layer flow in an incompressible viscous case under the effect of an applied magnetic field. Wahid [25] presented an exact solution of the Boltzmann kinetic equation with 4 collision frequency terms. The plasma flow characteristics of a rarefied electron gas generated by neutral atoms have been identified and explained in [27]. Further, they explained the behavior of the gas system but with an approximate solution and an inaccurate collision frequency formula. Abourabia and Tolba [37] investigated the behavior of a rarefied electron gas generated from noble gases using the method of moments in case of a discontinuous distribution function. They obtained an approximate solution for the shear stress and the mean flow velocity.

Furthermore, they considered that the immobile ions are kept as uniform and neutralizing background. Yan [38] designed a hybrid method with deviational particles using three methods (Monte Carlo, particle in cell, and macromicrodecomposition) for the inhomogeneous Vlasov-Poisson-Landau model in plasma. He divides the distribution function into a Maxwellian part generated by a grid-based fluid solver and a deviation part modeled by numerical deviational and coarse particles. A new model was proposed by Juno et al. [23] for the discretization of the kinetic VlasovMaxwell equations in a plasma with time using the FEM and Runge-Kutta method. They derived accurate solutions for the distribution function of the plasma. Pan et al. [19] used the charged particle transport by the electric field in the electric acceleration term of Boltzmann's equation in the BGK regime of the collision term. They discovered that, in discrete velocity space, once the nonequilibrium distribution function is produced, the probability distribution functions of the gas are discretized using the finite volume method. Using the discrete unified gas kinetic scheme, Liu et al. [39] developed a novel method for the Vlasov-Poisson equations with the BGK approximation at a wide range of $\mathrm{Kn}$ and Debye length. They simulated multiscale plasma using an asymptotic preserving scheme to investigate the electrical potential in all regimes. In our work, we obtain an exact solution for the model equations and study the problem thoroughly, considering the influence of electron-ion and electron-atom collisions in the Boltzmann equation collision term. In this study, we treat the complete collision frequencies of the Boltzmann kinetic equation and introduce a particular form of the model solution to avoid the discontinuity of the solutions. Also, we deal with the full values of variables without any cutoff caused by the small parameters' method implemented in [28]. The great advantage of our study is the consideration of the displacement current terms in the Maxwell equations, which are ignored in all mentioned papers previously (see [24-28, 37]), where they solved the differential equation system using this approximation, whereas in the current study, the term of the displacement current was taken into account because of the applied unsteady external magnetic field that gave this term great importance in our calculations. It cannot be ignored at all. The analytical results provided good agreement with the movement of helium plasma on a moving plate introduced by Wahid and Morad in Ref. [40].

The benefit of treating the Boltzmann kinetic equation enables us to consider the concepts of the linear nonequilibrium irreversible thermodynamics [10]. Besides, the use of Gibbs formulae with the distribution functions gives researchers the possibility to determine the entropy and entropy production of the plasma systems and illustrate the physical interpretation of the nonequilibrium thermodynamic properties $[10,38]$.

\subsection{The Physical Situation and Mathematical Formulation.} Assume that the upper half of the space at $y \geq 0$ is bounded by an illimitable plane plate at $y=0$. The space is filled with a plasma gas under the influence of an unsteady nonuniform external magnetic field $\left(B_{z \mathrm{E}}\right)$ normal to the direction of the flow. The plasma gas is initially in equilibrium. Considering this plasma in an applied magnetic field, we utilize the customary geometry. The electric field direction is along the $x$ -axis, and the applied magnetic field direction is along the $z$ -axis. The plane plate suddenly moves in its plane with a damping velocity $\left(V_{0} e^{-\alpha t}\right)$ along the $x$-axis.

Moreover, the plane plate is considered an insulator, uncharged, and impermeable. The system components (the plane plate +electrons + positive ions + neutral atoms) are kept at a constant temperature. We treated a frequency regime such that we can neglect ion currents compared to electron currents. Therefore, one can neglect the motion of ions and pay his attention to the electron motion.

The Lorentz force $\vec{F}_{e}$ acting on each electron can be acquired by the following:

$$
\vec{F}_{e}=-e \vec{E}-(e / c)(\vec{\xi} \wedge \vec{B})
$$

where $\vec{B}=B_{z} \vec{k}=\left(B_{z \mathrm{E}}+B_{z \mathrm{I}}\right) \vec{k}$, as $B_{z \mathrm{E}}=B_{0} e^{\phi(k y-\omega t)}$ 
Here, $B_{z \mathrm{E}}$ is the applied nonuniform unsteady external magnetic field, and $B_{z \mathrm{I}}$ is the induced magnetic field. They are functions of $y$, and $t$ while $\phi, \omega$, and $\kappa$ are constants. Here,

$$
\begin{gathered}
\vec{V} \equiv\left(V_{x}, 0,0\right), \\
\vec{J} \equiv\left(q n V_{x}, 0,0\right), \\
\vec{E} \equiv\left(E_{x}, 0,0\right), \\
\vec{B} \equiv\left(0,0, B_{z}\right),
\end{gathered}
$$

as $V_{x}, E_{x}, B_{z}$, and $J_{x}$ are functions of $y$ and $t$. In particular, this preference satisfies Maxwell's equations. In our model, the distribution function $\varphi_{e}(\vec{r}, \vec{\xi}, t)$ of the electrons in the plasma gas can be acquired by the Boltzmann kinetic equation that can be written in the BGK model in the form [5]

$$
\begin{aligned}
& \frac{\partial \varphi_{e}(\vec{r}, \vec{\xi}, t)}{\partial t}+\vec{\xi} \cdot \frac{\partial \varphi_{e}(\vec{r}, \vec{\xi}, t)}{\partial \vec{r}}+\frac{\vec{F}_{e}}{m_{e}} \cdot \frac{\partial \varphi_{e}(\vec{r}, \vec{\xi}, t)}{\partial \vec{\xi}} \\
& =\left(\frac{\varphi_{0 e}(\vec{r}, \vec{\xi}, t)-\varphi_{e}(\vec{r}, \vec{\xi}, t)}{\tau_{e e}}\right) \\
& +\left(\frac{\varphi_{0 i}(\vec{r}, \vec{\xi}, t)-\varphi_{e}(\vec{r}, \vec{\xi}, t)}{\tau_{e i}}\right)+\left(\frac{\varphi_{0 n}-\varphi_{e}}{\tau_{e n}}\right) \operatorname{as} \varphi_{\alpha 0} \\
& =n_{\alpha}\left(2 \pi R T_{\alpha}\right)^{-(3 / 2)} \exp \left(\frac{-\left(\vec{\xi}-\vec{V}_{\alpha}\right)^{2}}{2 R T_{\alpha}}\right)
\end{aligned}
$$

The primary arguments $n_{\alpha}, \vec{V}_{\alpha}$, and $T_{\alpha}$ that possess the character of the fundamental equations of motion can be obtained by taking moments of the kinetic equation for the distribution functions. Particles are reflected in full velocity accommodation from the plate, which means that the plasma particles are reflected from the plate with its velocity. Thus, the boundary conditions are [28] $V_{x 2}(0, t$ ) $=V_{0} e^{-\Omega t}$ for $t>0$, as $V_{x}=V_{x 2}$ as $\xi_{y}>0$, and $V_{x}$ is finite as $y \rightarrow \infty$.

Substituting from Equations (1) and (2)-(5) inside Equation (6), one gets

$$
\begin{aligned}
\frac{\partial \varphi_{e}}{\partial t}+ & \xi_{y} \frac{\partial \varphi_{e}}{\partial y}-\frac{e B_{e z}}{m_{e} c}\left(\xi_{y} \frac{\partial \varphi_{e}}{\partial \xi_{x}}-\xi_{x} \frac{\partial \varphi_{e}}{\partial \xi_{y}}\right)+\frac{e E_{e x}}{m_{e}} \frac{\partial \varphi_{e}}{\partial \xi_{x}} \\
& =\left(\frac{\varphi_{0 e}-\varphi_{e}}{\tau_{\mathrm{ee}}}\right)+\left(\frac{\varphi_{0 i}-\varphi_{e}}{\tau_{\mathrm{ei}}}\right)+\left(\frac{\varphi_{0 n}-\varphi_{e}}{\tau_{\mathrm{en}}}\right),
\end{aligned}
$$

as $\tau_{\mathrm{ee}}, \tau_{\mathrm{ei}}$, and $\tau_{\mathrm{en}}$ are electron-electron, electron-ion, and electron-neutral relaxation times, respectively, that are acquired by $[41,42]$

$$
\begin{aligned}
\tau_{\mathrm{ee}} & =\left(\frac{3 \sqrt{m_{e}} K_{B}^{3 / 2} T_{e}^{3 / 2}}{4 \sqrt{\pi} n_{e} e^{4} \log \left[\Lambda_{\mathrm{ee}}\right]}\right), \\
\tau_{\mathrm{ei}} & =\left(\frac{3 \sqrt{m_{e}} K_{B}^{3 / 2} T_{e}^{3 / 2}}{4 \sqrt{2 \pi} n_{i} e^{4} Z^{2} \log \left[\Lambda_{\mathrm{ei}}\right]}\right), \\
\tau_{\mathrm{en}} & =\left(\frac{3 m_{e} K_{B}^{3 / 2} T_{e}^{3 / 2}}{4 \sqrt{\pi m_{n}} n_{e} e^{4} \log \left[\Lambda_{\mathrm{en}}\right]}\right) .
\end{aligned}
$$

Here, $\log [\Lambda]=\log \left[4 \pi n \lambda_{D}^{3}\right]$ and $Z$ are the Coulomb Logarithm and the degree of ionization, respectively, and $\lambda_{D e}=$ $\lambda_{D i}=\lambda_{D}$ is the Debye length.

The pattern of the cone of influence suggested by Lee [42, 43] for the solution of the Boltzmann kinetic equation is used. The solution of Equation (7) can be written in the form

$$
\varphi=\left\{\begin{array}{l}
\varphi_{1}=n(2 \pi \mathrm{RT})^{-(3 / 2)}\left(1+\frac{\xi_{x} V_{x 1}}{\mathrm{RT}}\right) \exp \left(\frac{-\xi^{2}}{2 \mathrm{RT}}\right) \quad \text { for } \xi_{\mathrm{y}}<0 \downarrow \\
\varphi_{2}=n(2 \pi \mathrm{RT})^{-\frac{3}{2}}\left(1+\frac{\xi_{x} V_{x 2}}{\mathrm{RT}}\right) \exp \left(\frac{-\xi^{2}}{2 \mathrm{RT}}\right) \text { for } \xi_{\mathrm{y}}>0 \uparrow .
\end{array}\right.
$$

Here, $V_{x 1}$ and $V_{x 2}$ are two underdetermined functions of time $t$ and the single space variable $y$. Utilizing Grad's moment method in Ref. [7], multiplying Equation (7) by $\psi_{j}$ $(\vec{\xi})$, and integrating overall values of $\vec{\xi}$, we get the significant transfer equations for electrons in the following form:

$$
\begin{aligned}
& \frac{\partial}{\partial t} \int \psi_{j} \varphi_{e} d \underline{\xi}+\frac{\partial}{\partial y} \int \xi_{y} \psi_{j} \varphi_{e} d \underline{\xi}+\frac{e E_{x e}}{m_{e}} \int \varphi_{e} \frac{\partial \psi_{j}}{\partial \xi_{x}} d \underline{\xi}-\frac{e B_{z e}}{m_{e} c} \int \\
& \cdot\left(\xi_{x} \frac{\partial \psi_{j}}{\partial \xi_{y}}-\xi_{y} \frac{\partial \psi_{j}}{\partial \xi_{x}}\right) d \underline{\xi}=v_{\mathrm{ee}} \int\left(\varphi_{0 e}-\varphi_{e}\right) \psi_{j} d \underline{\xi} \\
& \quad+v_{\mathrm{ei}} \int\left(\varphi_{0 i}-\varphi_{e}\right) \psi_{j} d \underline{\xi}+v_{\mathrm{en}} \int\left(\varphi_{0 n}-\varphi_{e}\right) \psi_{j} d \underline{\xi}
\end{aligned}
$$

All integrals over the velocity dimension are evaluated using the relations below [7]:

$$
\int \psi_{j}(\vec{C}) \varphi d \underline{\xi}=\int_{-\infty}^{\infty} \int_{-\infty}^{0} \int_{-\infty}^{\infty} \psi_{j} \varphi_{1} d \underline{\xi}+\int_{-\infty}^{\infty} \int_{0}^{\infty} \int_{-\infty}^{\infty} \psi_{j} \varphi_{2} d \underline{\xi},
$$

where $\psi_{j}=\psi_{j}(\vec{\xi}), j=1,2$ and $d \underline{\xi}=d \xi_{x} d \xi_{y} d \xi_{z}$ and $\xi_{x}, \xi_{y}$ and $\xi_{z}$ are the particle velocities components along $x, y$, and $z$-axes, respectively. The electric and magnetic fields $E$ and $B$ can also get from Maxwell's equations for electrons as follows: 


$$
\begin{aligned}
& \frac{\partial E_{x e}}{\partial y}-\frac{1}{c} \frac{\partial B_{z e}}{\partial t}=0 \\
& \frac{\partial B_{z e}}{\partial y}-\frac{1}{c} \frac{\partial E_{x e}}{\partial t}-\frac{4 \pi e n_{e}}{c_{0}} V_{x e}=0 .
\end{aligned}
$$

Here, $n=\int \varphi d \underline{\xi}, n V_{x}=\int \xi_{x} \varphi d \underline{\xi}$, and the initial and boundary conditions

$$
\left.\begin{array}{l}
E_{x}(y, 0)=0, \\
B_{z}(y, 0)=B_{0}, \\
E_{x}(y, t), \\
B_{z}(y, t) \text { are finite as } y \rightarrow \infty .
\end{array}\right\}
$$

We offered the nondimensional variables defined by

$$
\left.\begin{array}{rl}
t & =t^{*} \tau_{\mathrm{ee}}, \\
y & =y^{*}\left(\tau_{\mathrm{ee}} c\right), \\
V_{x} & =V_{x}^{*} c, \\
\tau_{x y} & =\tau_{x y}^{*} c, \\
M & =\frac{V_{0}}{c}, \\
E_{x} & =E_{x}^{*}\left(\frac{m_{e} c}{e \tau_{\mathrm{ee}}}\right), \\
\rho & =n m, \\
B_{z} & =B_{z}^{*}\left(\frac{m_{e} c}{e \tau_{\mathrm{ee}}}\right), \\
\gamma & =\frac{m_{e}}{m_{i}}, \\
d U & =d U^{*}\left(K_{B} T_{e}\right), \\
\varphi_{j} & =\varphi_{j}^{*} n_{e}\left(2 \pi R T_{e}\right)^{-(3 / 2)}, \quad j=0,1,2 .
\end{array}\right\}
$$

The change in density and temperature is negligible for small values of Mach number, i.e., at $M^{2}=1$; therefore, we can assume that $n_{\alpha}=1+O\left(M^{2}\right)$ and $T_{\alpha}=1+O\left(M^{2}\right)$. Then, we can write the mean velocity and shear stress in the following form:

$$
\begin{aligned}
& V_{x}=\frac{1}{2}\left(V_{x 1}+V_{x 2}\right), \\
& \tau_{x y}=\frac{P_{x y}}{\rho V_{0} \sqrt{R T_{e} / 2 \pi}}=\left(V_{x 2}-V_{x 1}\right) .
\end{aligned}
$$

Here, $P_{x y}$ is defined by the relation $P_{x y}=m \int\left(\xi_{x}-V_{x}\right) \xi_{y}$ $\phi d \xi[42]$.
Utilizing the nondimensional variable, Equation (10) with $\psi_{1}=\xi_{x}$ and $\psi_{2}=\xi_{x} \xi_{y}$ becomes

$$
\begin{array}{r}
\frac{\partial V_{e x}^{*}}{\partial t^{*}}+\frac{\partial \tau_{e x y}^{*}}{\partial y^{*}}-E_{e x}^{*}=0 \\
\frac{\partial \tau_{e x y}^{*}}{\partial t^{*}}+2 \pi \frac{\partial V_{e x}^{*}}{\partial y^{*}}+\tau_{e x y}^{*}=0 .
\end{array}
$$

Moreover, the initial and boundary conditions take the form

$$
\left.\begin{array}{l}
V_{e x}^{*}\left(y^{*}, 0\right)=\tau_{e x y}^{*}\left(y^{*}, 0\right)=0, \\
2 V_{e x}^{*}\left(0, t^{*}\right)+\tau_{e x}^{*}\left(0, t^{*}\right)=2 M e^{-\varepsilon t^{*}}, \\
V_{e x}^{*} \text { and } \tau_{e x y}^{*} \text { are finite as } y \rightarrow \infty, \\
\varepsilon=w \tau_{\text {ee }} .
\end{array}\right\}
$$

Henceforth, for the sake of notational simplicity, we will drop the star on the non-dimensional variables. Thus, we have the next system of equations representing the boundary value problem for electrons:

$$
\begin{gathered}
\frac{\partial V_{e x}}{\partial t}+\frac{\partial \tau_{e x y}}{\partial y}-E_{e x}=0, \\
\frac{\partial \tau_{e x y}}{\partial t}+2 \pi \frac{\partial V_{e x}}{\partial y}+\left(1+\frac{\nu_{\mathrm{ei}}}{v_{\mathrm{ee}}}+\frac{\nu_{\mathrm{en}}}{v_{\mathrm{ee}}}\right) \tau_{e x y}=0, \\
\frac{\partial E_{e x}}{\partial y}-\frac{\partial B_{e z}}{\partial t}=0, \\
\frac{\partial B_{e z}}{\partial y}-\frac{\partial E_{e x}}{\partial t}-w_{e 0} V_{e x}=0, \quad w_{e 0}=\left(\frac{n_{e} e^{2}}{m_{e} v_{e e}^{2}}\right) .
\end{gathered}
$$

The traveling wave solution method [44-46] was utilized considering the new variable $\theta$ as

$$
\theta=k y-\omega t
$$

This procedure will transform the dependent variables as functions of the new variable $\theta$. Also, the transformation constants $k$ and $\omega$ can be measured from the boundary and initial conditions $[45,46]$.

The partial derivatives of Equations (19)-(22) can be determined from Equation (23) as follows:

$$
\begin{aligned}
\frac{\partial}{\partial t} & =-\omega \frac{\partial}{\partial \theta}, \\
\frac{\partial}{\partial y} & =k \frac{\partial}{\partial \theta}, \\
\frac{\partial^{n}}{\partial t^{n}} & =(-1)^{n} \omega^{n} \frac{\partial^{n}}{\partial \theta^{n}}, \\
\frac{\partial^{n}}{\partial y^{n}} & =k^{n} \frac{\partial^{n}}{\partial \theta^{n}}
\end{aligned}
$$


Substituting from Equations (23) and (24) into Equations (19)-(22), after various calculus manipulations, the obtained equations can be reduced to one equation:

$$
\begin{aligned}
& \left(\left(\omega^{2}-k^{2}\right)\left(\frac{2 \pi k^{2}}{\omega}-\omega\right)\right) \frac{d^{3} V_{e x}(\theta)}{d \theta^{3}}+w_{c}\left(\omega^{2}-k^{2}\right) \frac{d^{2} V_{e x}(\theta)}{d \theta^{2}} \\
& +w_{e 0} \omega \frac{d V_{e x}(\theta)}{d \theta}+w_{e 0} w_{c} V_{e x}(\theta)=0, \quad w_{c}=\left(1+\frac{v_{\mathrm{ei}}}{v_{\mathrm{ee}}}+\frac{v_{\mathrm{en}}}{v_{\mathrm{ee}}}\right) .
\end{aligned}
$$

The initial and boundary conditions after dropping the stars from the nondimensional variables are introduced:

$$
\left.\begin{array}{l}
E_{e x}(\theta=0)=\tau_{e x y}(\theta=0)=0, \\
B_{e z}(\theta=0)=B_{0}, \\
2 V_{e x}(\theta=-\omega)+\tau_{e x y}(\theta=-\omega)=2 M e^{-\varepsilon} \text { at } y=0, \text { e.g. } t=1, \\
V_{e x}, \tau_{e x y}, E_{e x}, \text { and } B_{e z} \text { are finite as } \theta \rightarrow-\infty .
\end{array}\right\}
$$

As a result of calculations on the model equations, we get the differential equation (25) with the conditions in Equation (26). This model equation is a third-order ordinary homogeneous differential equation that can be solved precisely with any symbolic software using the initial and boundary conditions Equation (26). When using these solutions, a more detailed description can be provided for the flows of laboratory argon plasma.

1.2. The Nonequilibrium Thermodynamic Investigations. The problem of the nonequilibrium thermodynamics of irreversible processes is fundamental when modeling any gas flow, and it continues to present considerable significance in the plasma dynamics. As a result of intensive research efforts in this field, scientists have found that the theoretical major of that theory and its applications in numerous branches of science starts from the thermodynamic laws and $H$-theorem essentials. Now, we can evaluate the entropy per unit mass $S$ in a nondimensional form as in the following relation (see, e.g., Refs. [24-26, 47, 48]):

$$
\begin{aligned}
S & =-\int \varphi_{e} \ln \varphi_{e} d \underline{\xi} \\
& =-\left(\int \varphi_{e 1} \ln \varphi_{e 1} d \underline{\xi}+\int \varphi_{e 2} \ln \varphi_{e 2} d \underline{\xi}\right) \\
& =-\pi^{\frac{3}{2}}\left[\left(V_{x 1}^{2}+V_{x 2}^{2}\right)-0.66\right] .
\end{aligned}
$$

As a consequence, we can get the entropy flux component in the $y$-direction:

$$
\begin{aligned}
J_{y}{ }^{(S)} & =-\int \xi_{y} \varphi_{e} \ln \varphi_{e} d \underline{\xi} \\
& =-\left(\int \xi_{y} \varphi_{e 1} \ln \varphi_{e 1} d \underline{\xi}+\int \xi_{y} \varphi_{e 2} \ln \varphi_{e 2} d \underline{\xi}\right) \\
& =\left[\pi\left({V_{x 1}}^{2}+V_{x 2}^{2}\right)\right] .
\end{aligned}
$$

From the entropy balance relation, one can define the entropy production in a local form $[47,48]$ :

$$
\sigma=\frac{\partial S}{\partial t}+\vec{\nabla} \cdot \overrightarrow{J^{(S)}}
$$

Taking into account the electromagnetic field energy, we can investigate the internal energy change of the entire system using the extended Gibbs formula [49] that includes the entire energy balance. The plasma gas magnetization's main types are paramagnetic and diamagnetic. Now, we can use the $1^{\text {st }}$ law of thermodynamics to formulate the total energy change for both kinds of magnetization. This includes the electromagnetic field energy balance as follows:

(i) In the paramagnetic case: the internal energy change can be written in terms of the entropy, $S$; polarization, $P$; and specific magnetization, $M$, which are the thermodynamic coordinates due to the temperature, $T$; electric field, $E$; and magnetic field, $B$, respectively. As a consequence, the extensive three participants of the internal energy change in the Gibbs relation: $d U=d U_{S}+d U_{\text {pol }}+d U_{\text {para }}$, in which $d U_{S}=T d S$ is the internal energy change according to the change in entropy. $d U_{\mathrm{pol}}=E d P$ is the internal energy change according to change in polarization. Moreover, $d U_{\text {para }}=B d M$ is the internal energy change according to the change in magnetization, where $M$ is calculated from the equation $[37,49]: \partial$ $S / \partial M_{B}=-(B / T) \Rightarrow M_{B}=-\int((T / B)(\partial S / \partial y))_{t} d y$.

Using the nondimensional variables $U^{*}=U / m_{e} c^{2}$, $M_{B}^{*}=M_{B}\left(\nu_{\mathrm{ee}} / e c\right)$, and $p^{*}=p\left(\nu_{\mathrm{ee}} / e c\right)$ in the Gibbs relation, we can get (after dropping the star) $d U=$ $d S_{e}+f_{1} E_{x} d p+f_{1} B_{z} d M_{B}$

(ii) In the diamagnetic case: the internal energy change can be written in terms of the extensive thermodynamic quantities $S$ and $P$ and the induced magnetic field, $B$, which symbolize the thermodynamic coordinates due to the intensive quantities $T, E$, and $M_{B}$, respectively; thus, we have three participants in the internal energy modified in the Gibbs formula acquired by

$$
d U=d U_{S}+d U_{\mathrm{pol}}+d U_{\mathrm{dia}}
$$

as $d U_{\text {dia }}=-M_{B} d B$ is the internal energy change according to the change in the generated magnetic field induction, as $M_{B}$ $=T(\partial S / \partial B)[37,49]$. In the present case, the internal energy $d U$, in a nondimensional form, is written in the compact form as $d U=d S+E d p-M_{B} d B$ and $d S=(\partial S / \partial r) \delta y+(\partial S / \partial t) \delta t$.

\section{Discussion}

In the present study, we have investigated the unsteady manner of plasma gas based on the Boltzmann's kinetic theory and irreversible thermodynamics using the Boltzmann equation's exact traveling wave solution with the accurate values 

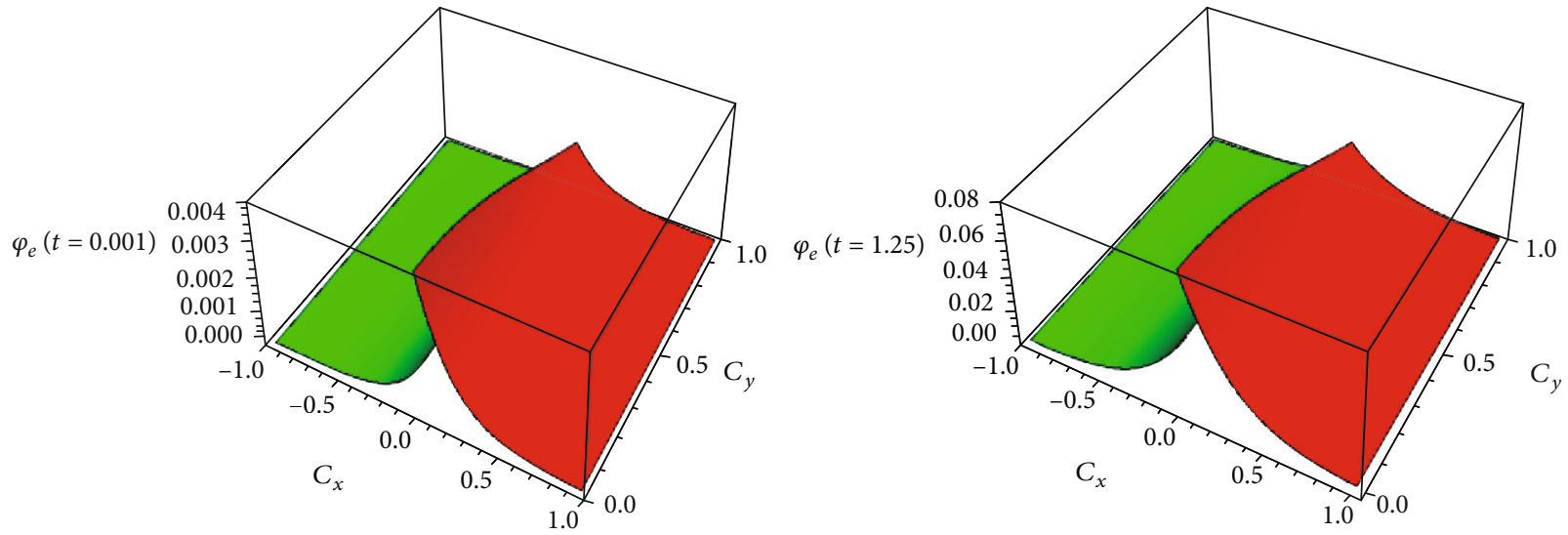

(a)
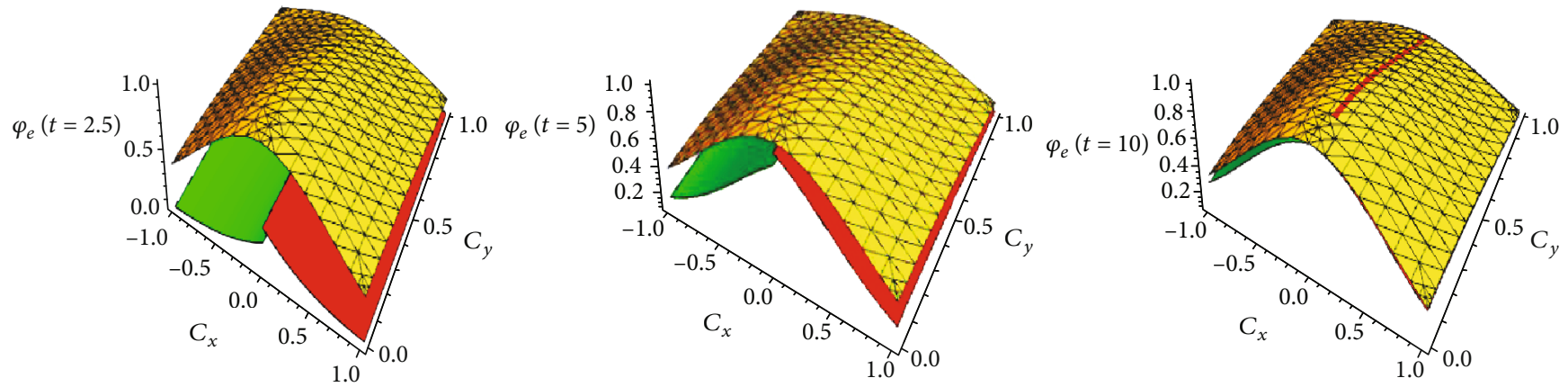

(b)

FIgURE 1: (a) The perturbed electron velocity distribution functions $\varphi_{e}\left[\varphi_{1}\right.$ (green), $\varphi_{2}$ (red) $]$ at $(t=0.001$ and 1.25$)$ for a fixed $y$ value $(y=1)$ with the Mach number of the plate $M=0.01$. (b) The combination of the perturbed electron velocity distribution functions $\varphi_{e}: \varphi_{1}$ (green) and $\varphi_{2}$ (red) compared to the equilibrium velocity distribution function $\varphi_{\mathrm{o}}$ (grid) at $t=2.5,5$, and 10 with the Mach number of the plate $M=0.01$ at $y=1$.

of electron-electron, electron-ion, and electron-atom collision frequencies in the BGK technique of the collision terms. The results are clarified in figures for dilute plasma argon gas. It is shown that the computations due to the methods of solution are well performed using standardized data for electron gas in an argon plasma. The analytical results fit very well with the plasma laboratory $[28,41]$ in the following two cases: in the paramagnetic medium, based on the ionizing potential of the atoms, the argon gas loses single electrons, while in the diamagnetic case, the argon gas loses electron pairs.

We present the solutions of the model equation using the idea of the shooting numerical calculation method to estimate the transformation constants $k=0.85$ and $\omega=0.1$ and the Mach number of the plate $M=1.0 \times 10^{-2}$ as presented in [15]. The calculations are carried out for the problem according to the following plasma fluid properties and conditions: Boltzmann constant, initial temperature, electron concentration, and diameter of the argon atom are given by $k_{B}=1.3807 \times 10^{-16} \mathrm{erg} / \mathrm{K}, T_{0}=200 \mathrm{~K}, n_{e}=10^{17} \mathrm{~cm}^{-3}$, and $d=$ $3.84 \times 10^{-8} \mathrm{~cm}$. The electron rest mass and electron charge are given by $m_{e}=9.093 \times 10^{-28} \mathrm{~g}$ and $e=4.810^{-10} \mathrm{esu}$, which are used to determine the dimensionless control parameter $\alpha_{0}=1.6 \times 10^{-12}$. Besides, the electron-ion, electron-electron, and electron-neutral atom collision frequency values are $v_{\mathrm{ei}}$
$=1.46 \times 10^{15} \mathrm{sec}^{-1}, v_{\mathrm{ee}}=1.033 \times 10^{15} \mathrm{sec}^{-1}$, and $v_{\mathrm{en}}=1.199$ $\times 10^{4} \mathrm{sec}^{-1}$, respectively. Finally, the plasma's mean free path $\lambda=\left(\sqrt{2} \pi n_{e} d^{2}\right)^{-1}=1.526 \times 10^{-3} \mathrm{~cm}$, which is large compared with one of the most fundamental properties, which is the electron Debye length $\lambda_{D e}=\sqrt{K_{B} T_{0} / 4 \pi n_{e} e^{2}}=3.086$ $\times 10^{-7} \mathrm{~cm}$.

The behavior of solutions that appear in Figures 1(a) and 1(b) reveals that the effect of the plate motion on the electron velocity distribution function is very considerable. Figure 1(b) displays the perturbed electron velocity distribution function in the nearby area of the flat plate that suddenly sets in motion with Mach number equals to 0.01. As shown in Figure 1(b), the deviation from equilibrium decreases with time as the system tries to reach an equilibrium state as expected from Le Chatelier's principle. Therefore, the electron velocity distribution function $F_{e}$ approaches to equilibrium velocity distribution function $F_{0}$ as $t=10$, a result that strengthens the interpretation of the equilibrium principle. According to Le Chatelier, the position of equilibrium at a certain point in time for the perturbed electron velocity distribution functions $F_{1}$ and $F_{2}$ approaches to the equilibrium distribution function $F_{0}$, which is of interest to our problem (see Figure 1(b)).

Figures 2-6 show, respectively, the graphics of the velocity, shear stress, viscosity, applied magnetic field, and the 


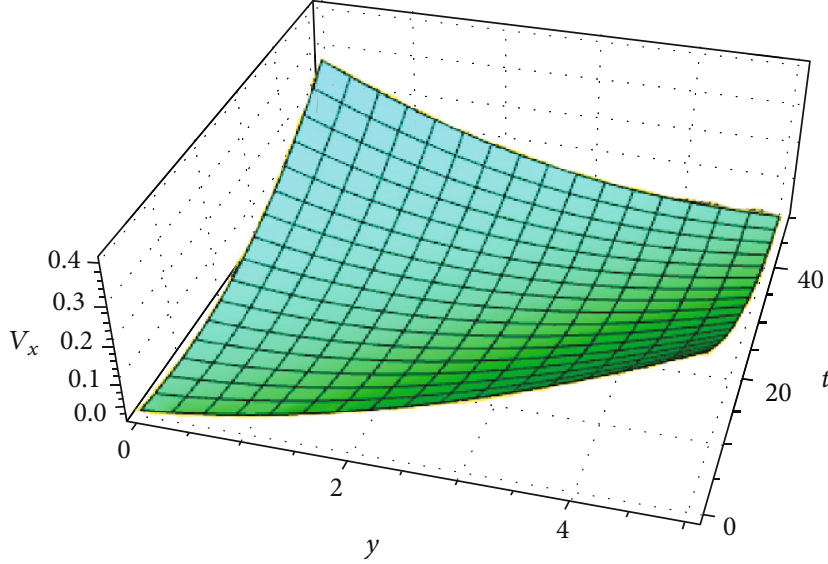

Figure 2: Spatiotemporal velocity profile $V_{x}$.

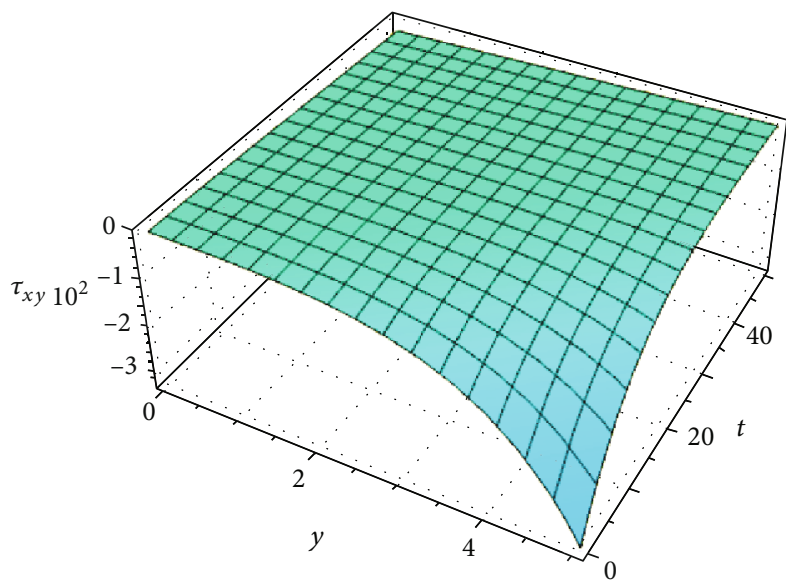

Figure 3: The shear stress $\tau_{x y}$ vs. space $y$ and time $t$.

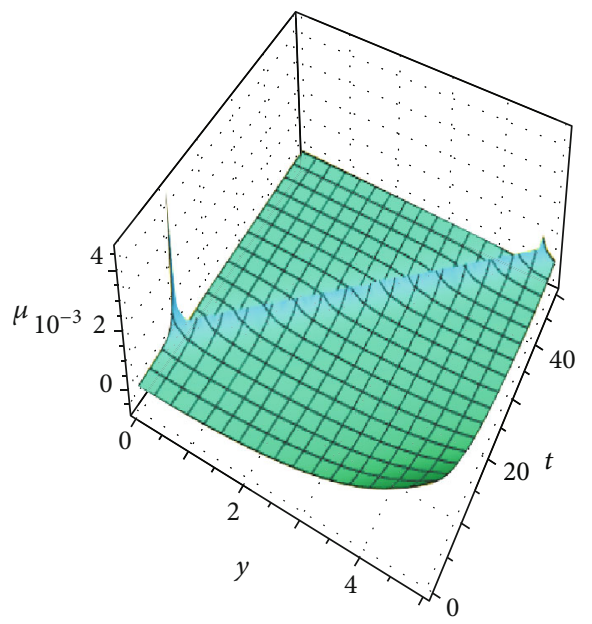

FIGURE 4: The viscosity coefficient $\mu$ vs. space $y$ and time $t$.

induced electric field according to it. We see that near the moving plate, the mean velocity of the electrons in Figure 2 has a value equal to Mach number $M=0.01$ of the flat plate as $(y, t)=(0,0)$. It decreases exponentially with time, which gives a good agreement with the results presented in [28,

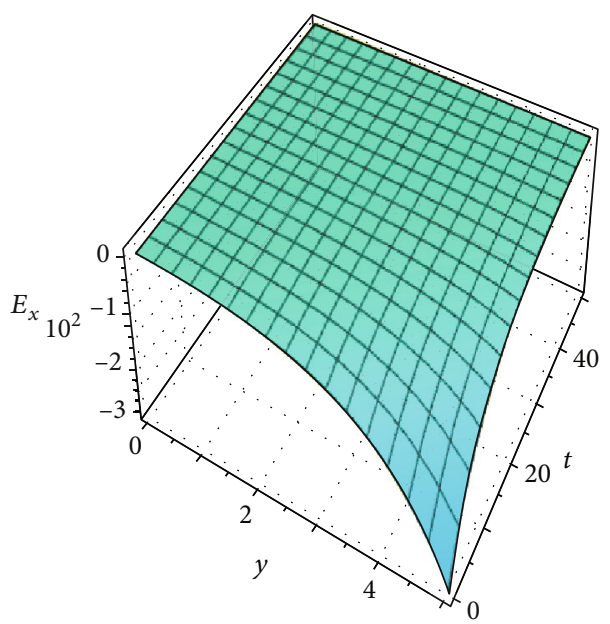

Figure 5: The induced electric field $E_{x}$ vs. space $y$ and time $t$.

37]. The shear stress decreases with time, as shown in Figure 3 . The viscosity coefficient follows the equilibrium law by counteracting the change such that the system tends to equilibrate with increasing viscosity, as seen in Figure 4. We shed light upon the behavior of the generated electric and applied magnetic fields in Figures 5 and 6 . They illustrate that the electric and magnetic fields are decreasing in magnitudes that tend to zero value over time. That is due to our assumption of the applied external magnetic field and due to the dependency of the generated electric field to the external magnetic field as they connected by Maxwell's equations. Away from the plate, the generated electric field increases with time (see Figure 5). However, the magnetic field has the maximum value at the beginning of time, and then, it decreases nonlinearly towards zero for all $y$ values, as shown in Figure 6. In such a way, the effects of the electromagnetic fields are dominant compared with dynamic effects in the plasma flow model.

From the results for velocity, density, and temperature, which were obtained from the solution of the mathematical model, we can demonstrate the nonlinear behavior of the entropy, as seen in Figure 7. The entropy $S$ increases for argon plasma with time, which gives a good agreement with the $2^{\text {nd }}$ law of thermodynamics [38]. The entropy production $\sigma$ decreases once the system goes towards the equilibrium till it reaches the state of equilibrium, i.e., $\sigma=0$ and $S$ is maximum (see Figures 7 and 8). The entropy production of the plasma model satisfies the fundamental laws of thermodynamics and the Boltzmann $H$-theorem as $\sigma \geq 0$ for all values of the position $y$ and the time $t$, as shown in Figure 8 .

As shown in Figure 9, the change in the internal energy due to the variation of entropy nonlinearly decreases with time and space. For the paramagnetic plasma cases of $d U_{\mathrm{pol}}=E d P$ and $d U_{\text {para }}=B d M$, the internal energy changes are plotted in Figures 10 and 11 . We note that the internal energy polarization and magnetization change is nonlinearly increased.

An investigation of the stability requires to study the time rate of change (time derivatives) of the internal energy $U\left(\lim _{\Delta t \rightarrow 0}(\Delta U / \Delta t)=\lim _{\Delta t \rightarrow 0}\left(\Delta U_{S} / \Delta t\right)+\lim _{\Delta t \rightarrow 0}\left(\Delta U_{\mathrm{pol}} / \Delta t\right)+\lim _{\Delta t \rightarrow 0}(\Delta\right.$ 


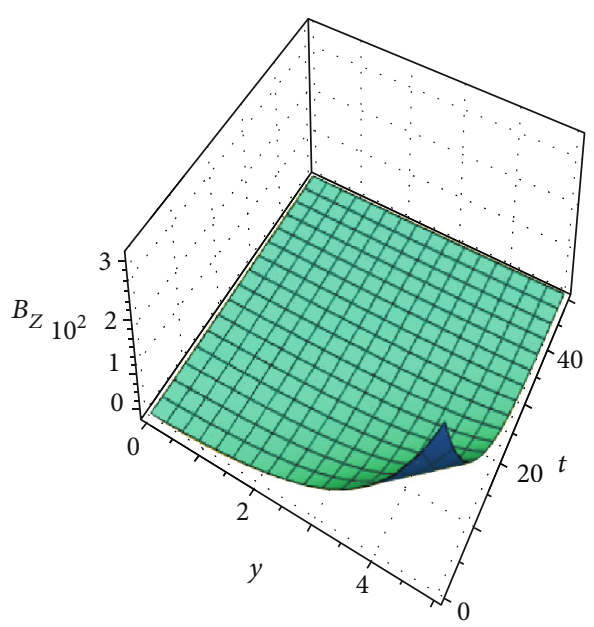

Figure 6: The applied magnetic field $B_{z}$ vs. space $y$ and time $t$.

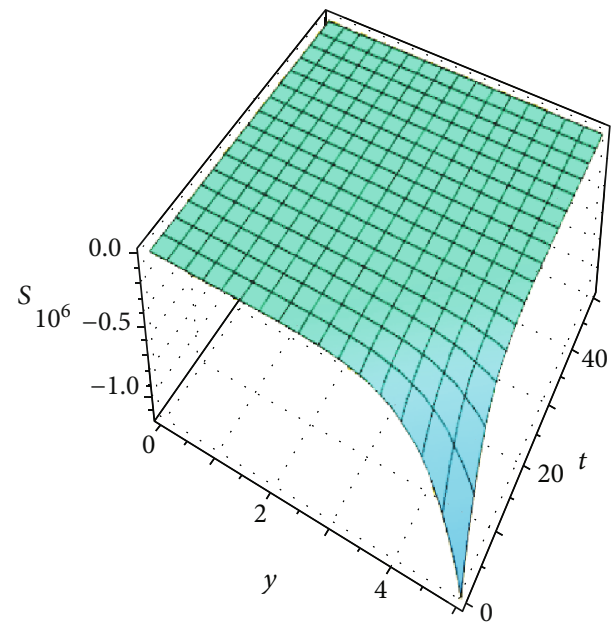

Figure 7: The spatiotemporal entropy profile $S$.

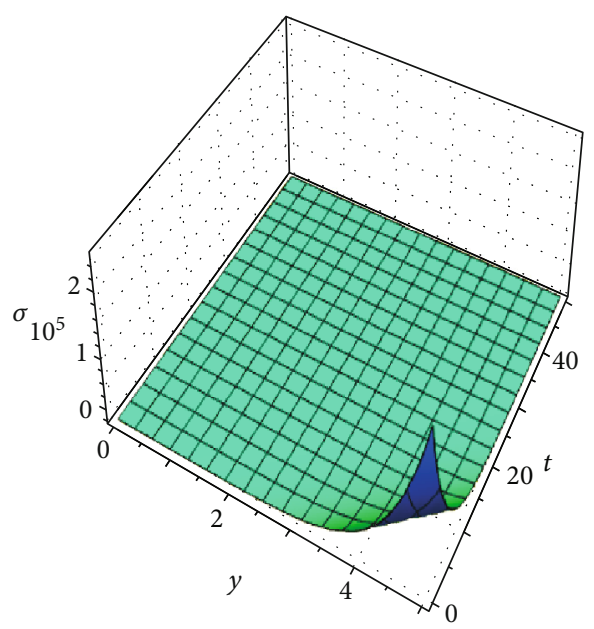

FIGURE 8: The entropy production $\sigma$ vs. space $y$ and time $t$.

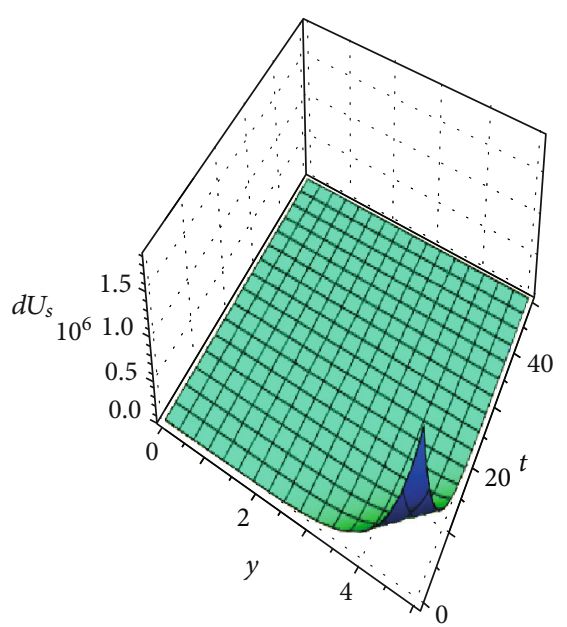

Figure 9: The internal energy change $d U_{S}$ vs. space $y$ and time $t$.

$\left.\left.U_{\text {para }} / \Delta t\right)\right)$. The right-hand side terms can be positive, negative, or null. The positive values indicate an increase in time of internal energy, whereas negative values indicate a decrease in internal energy. On the other side, if the sum of the terms is equal to null, then the rate of change in the internal energy vanishes. Besides, the necessary and sufficient condition for the system to be in the equilibrium state is $\sigma$ $=0$ and $d U=0$, which is indicated in Figures 8-11.

The electron gyrofrequency, which is the angular frequency of the circular motion of a charged particle in the plane perpendicular to the applied magnetic field, gradually decreases with time for argon plasma and increases away from the plate (see Figure 12), whereas in plate vicinity, electron Larmor radius or gyroradius, which is the radius of the circular motion of an electron in the plane perpendicular to the applied magnetic field, has the maximum value for argon. Then, it vanishes away from the vicinity of the plate, as shown in Figure 13.

\section{Conclusions}

In this paper, a detailed calculation of the effect of the collisions of the electrons with positive ions and neutral atoms operating with argon plasma has been made. Further, the current displacement term in the Maxwell equations is taken into consideration, which was ignored in the previous works (see [24-28, 37]). We have elaborated a model to calculate the distribution functions, velocities, and electromagnetic fields of the plasma flow under the effect of an external magnetic field.

All graphics presented in the text have shown excellent agreement within the investigated ranges of the governing parameters and initial and boundary conditions. This fact supports the following idea: based on the BGK model, the collisionless plasma flow problem can be studied in the framework of the Boltzmann kinetic equation supplemented by the Maxwell equations. Also, we can investigate the behavior of electron gas, which is generated from noble gases under the effect of a nonuniform unsteady external magnetic field using the method of moments of the kinetic equation for 


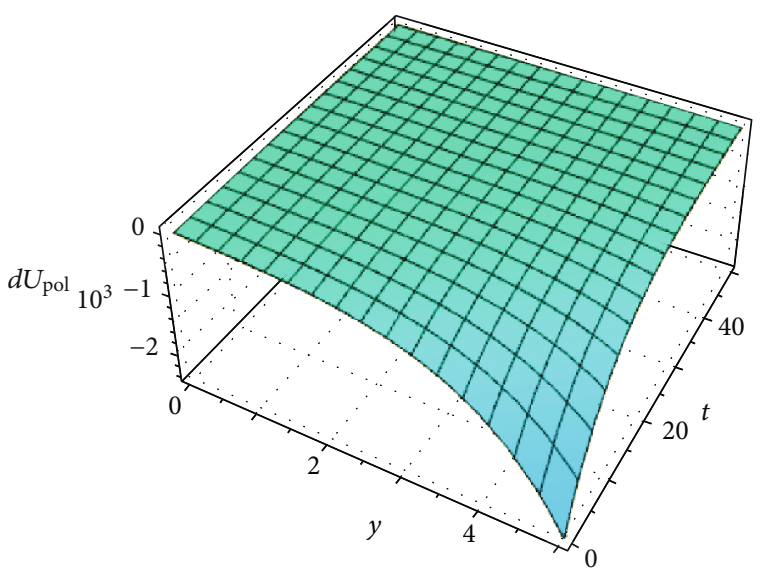

FIGURE 10: The internal energy change $d U_{\text {pol }}$ vs. space $y$ and time $t$.

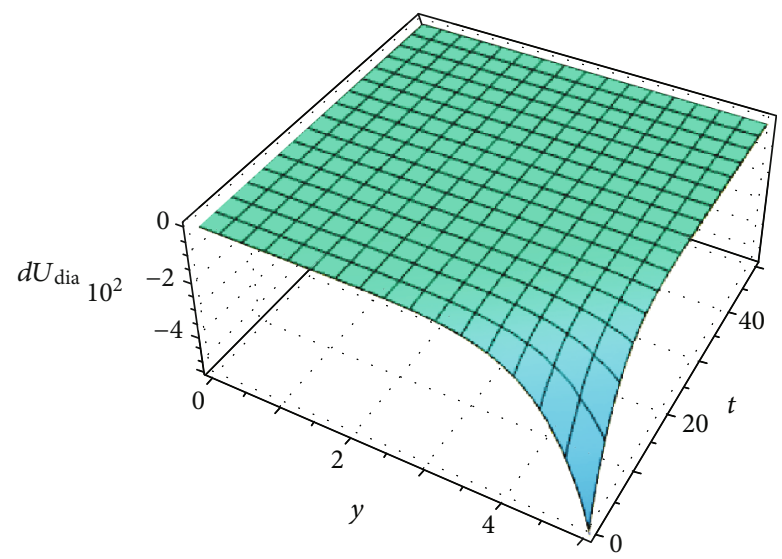

FIGURE 11: The internal energy change $d U_{\text {dia }}$ vs. space $y$ and time $t$.

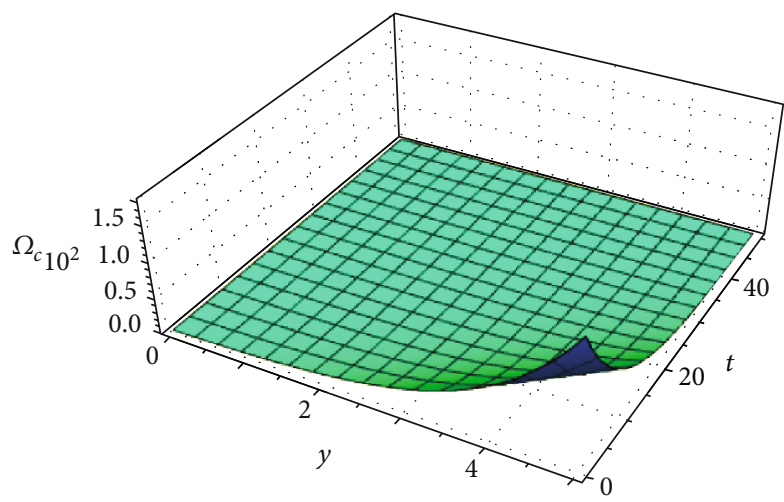

FIGURE 12: The gyrofrequency $\Omega_{c}$ vs. space $y$ and time $t$.

the two-sided distribution functions of the velocity. From a thermodynamic viewpoint, collisions between plasma components (ions, electrons, and atoms) are characterized by the fluctuation between loss and gain part of the energy of the charged particles (electrons).

The calculated distribution functions are used to estimate entropy and entropy production that are compatible with the nonequilibrium thermodynamic laws. These properties are

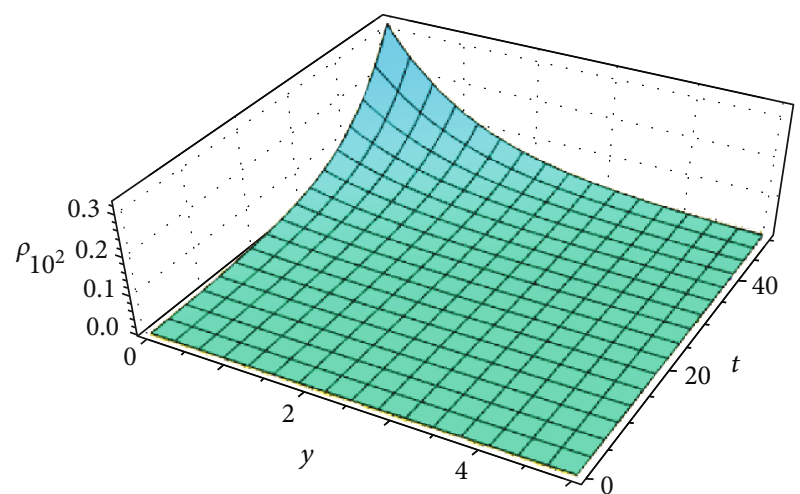

FIGURE 13: The gyroradius $\bigotimes$ versus space $y$ and time $t$.

deeply discussed. After drawing the solutions, a good consistency between them was found with the thermodynamic laws. Finally, it should be added that the discovered behavior of the collision processes between electrons and ions in argon plasma based on the BGK technique of the Boltzmann kinetic equation might depend on the velocity of the moving plate in the rarefied gas dynamics. This point has been examined minutely in this paper.

\section{Data Availability}

The data used to support the findings of this study are included within the article.

\section{Conflicts of Interest}

The authors declare that there is no conflict of interest regarding the publication of this paper.

\section{Acknowledgments}

This study is supported by the Egyptian Academy of Scientific Research and Technology by the associated grant number (No. 6508), under the program of Science UP Faculties of Science.

\section{References}

[1] C. G. N. Lee, K. J. Kanarik, and R. A. Gottscho, "The grand challenges of plasma etching: a manufacturing perspective," Journal of Physics D: Applied Physics, vol. 47, no. 27, p. 273001, 2014.

[2] V. Miller, A. Lin, and A. Fridman, "Why target immune cells for plasma treatment of cancer," Plasma Chemistry and Plasma Processing, vol. 36, no. 1, pp. 259-268, 2016.

[3] K. Weltmann, J. Kolb, M. Holub et al., "The future for plasma science and technology," Plasma Processes and Polymers, vol. 16, article e1800118, 2019.

[4] G. Belmont, R. Grappin, F. Mottez, F. Pantellini, and G. Pelletier, Collisionless Plasmas in Astrophysics, WILEYVCHVerlag GmbH \& Co. KGaA, Boschstr, Weinheim, Germany, 2014.

[5] P. L. Bhatnagar, E. P. Gross, and M. Krook, "A model for collision processes in gases. I. Small amplitude processes in 
charged and neutral one-component systems," Physical review, vol. 94, no. 3, pp. 511-525, 1954.

[6] Z. Donko and N. Dyatko, "First-principles particle simulation and Boltzmann equation analysis of negative differential conductivity and transient negative mobility effects in xenon," European Physical Journal D: Atomic, Molecular, Optical and Plasma Physics, vol. 70, no. 6, p. 135, 2016.

[7] H. Struchtrup, "Grad's moment method," in Macroscopic Transport Equations for Rarefied Gas Flows, Interaction of Mechanics and Mathematics, Springer, Berlin, Heidelberg, 2005.

[8] A. M. Abourabia and T. Z. A. Wahid, "Solution of the Krook kinetic equation model and non-equilibrium thermodynamics of a rarefied gas affected by a nonlinear thermal radiation field," Journal of Non-Equilibrium Thermodynamics, vol. 36, pp. 75-98, 2011.

[9] A. M. Abourabia and T. Z. A. Wahid, "Kinetic and thermodynamic treatment for the Rayleigh flow problem of an inhomogeneous charged gas mixture," Journal of Non-Equilibrium Thermodynamics, vol. 37, no. 1, pp. 1-25, 2012.

[10] T. A. Wahid, Kinetic Irreversible Thermodynamic Study of Plasma and Neutral Gases, LAP LAMBERT Academic Publishing, Germany, 2014.

[11] V. Shahabi, T. Baier, E. Roohi, and S. Hardt, "Thermally induced gas flows in ratchet channels with diffuse and specular boundaries," Scientific Reports, vol. 7, no. 1, p. 41412, 2017.

[12] J. Zhao, J. Yao, M. Zhang et al., "Study of gas flow characteristics in tight porous media with a microscale lattice Boltzmann model," Scientific Reports, vol. 6, no. 1, p. 32393, 2016.

[13] H. Xiao and K. Tang, "A unified framework for modeling continuum and rarefied gas flows," Scientific Reports, vol. 7, no. 1, p. 13108, 2017.

[14] B.-Y. Cao, J. Sun, M. Chen, and Z.-Y. Guo, "Molecular momentum transport at fluid-solid interfaces in MEMS/NEMS a review," International Journal of Molecular Sciences, vol. 10, no. 11, pp. 4638-4706, 2009.

[15] T. Z. A. Wahid, "Kinetic and thermodynamic treatment for the exact solution of the unsteady Rayleigh flow problem of a rarefied homogeneous charged gas," Journal of Non-Equilibrium Thermodynamics, vol. 37, no. 2, pp. 119-141, 2012.

[16] H. A. Tighchi, M. Sobhani, and J. A. Esfahani, "Effect of volumetric radiation on natural convection in a cavity with a horizontal fin using the lattice Boltzmann method," The European Physical Journal Plus, vol. 133, no. 1, p. 8, 2018.

[17] M. Barzegar Gerdroodbary, D. D. Ganji, M. Taeibi-Rahni, and S. Vakilipour, "Effect of Knudsen thermal force on the performance of low-pressure micro gas sensor," The European Physical Journal Plus, vol. 132, no. 7, p. 315, 2017.

[18] M. Lesur, "Method- and scheme-independent entropy production in turbulent kinetic simulations," Computer Physics Communications, vol. 200, pp. 182-189, 2016.

[19] D. Pan, C. Zhong, C. Zhuo, and W. Tan, "A unified gas kinetic scheme for transport and collision effects in plasma," Applied Sciences, vol. 8, no. 5, p. 746, 2018.

[20] K. Tang and H. Xiao, "Entropy conditions involved in the nonlinear coupled constitutive method for solving continuum and rarefied gas flows," Entropy, vol. 19, no. 12, p. 683, 2017.

[21] L. Ferrari, "Comparing Boltzmann and Gibbs definitions of entropy in small systems," The European Physical Journal Plus, vol. 132, no. 11, p. 487, 2017.
[22] D. Kalempa and F. Sharipov, "Sound propagation through a binary mixture of rarefied gases at arbitrary sound frequency," European Journal of Mechanics-B/Fluids, vol. 57, pp. 50-63, 2016.

[23] J. Juno, A. Hakim, J. TenBarge, E. Shi, and W. Dorland, "Discontinuous Galerkin algorithms for fully kinetic plasmas," Journal of Computational Physics, vol. 353, pp. 110-147, 2018.

[24] T. Z. A. Wahid and S. K. Elagan, "Kinetic treatment for the exact solution of the unsteady Rayleigh flow problem of a rarefied homogeneous charged gas bounded by an oscillating plate," Canadian Journal of Physics, vol. 90, pp. 987-998, 2012.

[25] T. Z. A. Wahid, "Travelling waves solution of the unsteady flow problem of a rarefied nonhomogeneous charged gas bounded by an oscillating plate," Mathematical Problems in Engineering, vol. 2013, Article ID 503729, 13 pages, 2013.

[26] A. M. Abourabia and T. Z. A. Wahid, "Kinetic and thermodynamic treatments of a neutral binary gas mixture affected by a nonlinear thermal radiation field," Canadian Journal of Physics, vol. 90, no. 2, pp. 137-149, 2012.

[27] T. Z. A. Wahid, "Travelling wave solution of the unsteady BGK model for a rarefied gas affected by a thermal radiation field," Sohag Journal of Mathematics, vol. 2, no. 2, pp. 75-87, 2015.

[28] T. Z. A. Wahid, "Travelling waves solution of the unsteady flow problem of a collisional plasma bounded by a moving plate," Fluid Mechanics, vol. 4, no. 1, pp. 27-37, 2018.

[29] L. Oliva, "Impact of the pre-equilibrium stage of ultrarelativistic heavy ion collisions: isotropization and photon production," The European Physical Journal Plus, vol. 134, no. 6, p. 306, 2019.

[30] P. Ma, Y. Zheng, and G. Qi, "The nonextensive Bose-Einstein condensation and photon gas with parameter transformation," The European Physical Journal Plus, vol. 134, no. 10, p. 502, 2019.

[31] A. M. Abourabia and A. M. Morad, "Exact traveling wave solutions of the van der Waals normal form for fluidized granular matter," Physica A, vol. 437, pp. 333-350, 2015.

[32] Ž. Mladenović, S. Gocić, D. Marić, and Z. L. Petrović, "Influence of space charge density on electron energy distribution function and on composition of atmospheric pressure He/O2/air plasmas," The European Physical Journal Plus, vol. 133, no. 8, p. 344, 2018.

[33] T. Z. A. Wahid, "Exact solution of the unsteady Krook kinetic model and nonequilibrium thermodynamic study for a rarefied gas affected by a nonlinear thermal radiation field," Canadian Journal of Physics, vol. 91, no. 3, pp. 201-210, 2013.

[34] W. Marques and A. R. Méndez, "On the kinetic theory of vehicular traffic flow: Chapman-Enskog expansion versus Grad's moment method," Physica A, vol. 392, no. 16, pp. 3430-3440, 2013.

[35] G. M. Kremer, An Introduction to the Boltzmann Equation and Transport Processes in Gases, Springer-Verlag, Berlin Heidelberg, 2010.

[36] T. S. Chang and C. M. Chang, "Rayleigh's problem in collisionless plasmas," Plasma Physics, vol. 13, no. 9, pp. 695-714, 1971.

[37] A. M. Abourabia and R. E. Tolba, "On the irreversible thermodynamics of an electron gas in the vicinity of a suddenly moving rigid plate," The European Physical Journal Plus, vol. 127, no. 58, pp. 1-11, 2012.

[38] B. Yan, "A hybrid method with deviational particles for spatial inhomogeneous plasma," Journal of Computational Physics, vol. 309, no. 18-36, pp. 18-36, 2016. 
[39] H. Liu, F. Shi, J. Wan, X. He, and Y. Cao, "Discrete unified gas kinetic scheme for a reformulated BGK-Vlasov-Poisson system in all electrostatic plasma regimes," Computer Physics Communications, vol. 255, no. 107400, p. 107400, 2020.

[40] T. Z. A. Wahid and A. M. Morad, "Unsteady plasma flow near an oscillating rigid plane plate under the influence of an unsteady nonlinear external magnetic field," IEEE Access, vol. 8, pp. 76423-76432, 2020.

[41] J. D. Huba, NRL Plasma Formulary, Naval Research Laboratory, Washington, DC, 2019.

[42] S. I. Braginskii, Transport processes in a plasma. Reviews of Plasma Physics, Volume 1. Authorized translation from Russian by Herbert Lashinsky, University of Maryland, USA, M. A. Leontovich, Ed., Published by Consultants Bureau, New York, 1965.

[43] L. Lees, "Kinetic theory description of rarefied gas flow," Journal of the Society for Industrial and Applied Mathematics, vol. 13, no. 1, pp. 278-311, 1965.

[44] A. M. Abourabia, T. S. El-Danaf, and A. M. Morad, "Exact solutions of the hierarchical Korteweg-de Vries equation of microstructured granular materials," Chaos, Solitons \& Fractals, vol. 41, no. 2, pp. 716-726, 2009.

[45] J. Gratton, S. M. Mahajan, and F. Minotti, Non-Newtonian gravity creeping flow, International Centre for Theoretical Physics, Trieste (Italy), 1988.

[46] G. Nugroho, A. M. S. Ali, and Z. A. Abdul Karim, "Towards a new simple analytical formulation of Navier-Stokes equations," World Academy of Science, Engineering and Technology, vol. 39, pp. 197-201, 2009.

[47] G. Lebon, D. Jou, and J. Casas-Vàzquez, Understanding Nonequilibrium Thermodynamics: Foundations, Applications, Frontiers, Springer-Verlag, Berlin, Heidelberg, Germany, 2008.

[48] T. Z. Abdel Wahid, The Effect of Lorentz and Centrifugal Forces on Gases and Plasma, LAMBERT Academic Publishing, Germany, 2017.

[49] P. Van der Linde, "Thermodynamic stability of dia-and paramagnetic materials," Periodica Polytechnica Chemical Engineering, vol. 12, p. 97, 1998. 